hohen Temperaturen, wurden die charakteristischsten Beispiele in einem Film festgehalten.

Max-Planck-Institut für Silikatforschung, Würzburg

Eingegangen am 18. Mai 1960

R. BRÜCKNER

1) BRÜCKNER, R.: Naturwissenschaften 47, 371 (1960). 2) SrgWart, K., u. H. NAsSENSTEIN : Naturwiss. 42, 458 (1955). 3) Kroepelin, H., u. H. G. NeumanN: Naturwissenschaften 43, 347 (1956); 44, 304 (1957); 45, 333 (1958).

\section{In Relation with Surface Tension}

By studying surface tension, one of us has discovered the following new effect ${ }^{3 a}$ ): in a domain of temperature far from the boiling point, by putting a pure liquid into rotation, the value of surface tension becomes different from the value in rest. For convenience we have called this phenomenon rotationalkinetic effect (r. e.). In this domain the surface tension against temperature curve is situated generally above the rest values, and presents, in a little interval, a minimum which in the case of some liquids descends under the straight line known in the literature ${ }^{4}$ ). In other words, in this interval of temperature the difference between the values of surface tension of the rotating liquid and the rest values is less, or even negative. The phenomenon has been studied in several works $\left.\left.\left.{ }^{1}\right), 2 a, b, e, 1\right), 3 b\right)$.

At temperatures nearer the boiling point $r$. e. does not $\operatorname{appear}^{2 \mathrm{c}}$.

The whole experimental study has been performed by the ring method. A verification of the existence of $r$. e. has been made with another method. This was based upon the counting of bubbles raising from the end of a tube introduced vertically in the liquid, through which one pumps air in known quantities ${ }^{2 d}$ ). The greater the surface tension is, the less the number of bubbles. With this method the existence of r. e. at low temperatures, and its disappearance at high temperatures has been confirmed.

In the present paper we communicate the results obtained similarly by the bubbles method, which put in evidence the minimum. The rotation frequency has been $100 \mathrm{rev} / \mathrm{min}$ on this occasion (Table).

Table. Number of bubbles

\begin{tabular}{c|c|c|c|c|c|c|c}
\hline Liquid & Temp. $\left({ }^{\circ} \mathrm{C}\right)$ & $\left.\mathrm{I}^{*}\right)$ & $\left.\mathrm{II}{ }^{*}\right)$ & Liquid & Temp. $\left({ }^{\circ} \mathrm{C}\right)$ & $\mathrm{I}$ & II \\
\hline \multirow{2}{*}{ Benzene } & $11,1-13,0$ & 410 & 375 & Glycol & $14,0-15,1$ & 293 & 275 \\
& $14,5-15,8$ & 412 & 374 & & $18,4-19,2$ & 304 & 295 \\
& $16,0-17,3$ & 420 & 420 & & $19,7-20,7$ & 306 & 315 \\
& $20,0-22,1$ & 424 & 385 & & $21,0-21,5$ & 319 & 317 \\
& & & & & $25,0-25,6$ & 337 & 314
\end{tabular}

*) Number of bubbles $( \pm 2 \%)$ : I rest, II rotation.

One observes the existence of the minimum aproximately between $16^{\circ} \mathrm{C}$ and $18^{\circ} \mathrm{C}$ in the case benzene, and between $19^{\circ} \mathrm{C}$ and $22^{\circ} \mathrm{C}$ in the case of glycol. Taking account of the rotation frequency, it is clear that the above results are according to those obtained by the ring method $\left.{ }^{2 b}, \mathrm{c}\right)$.

We have shown ${ }^{2 d}$ ) that the appearance of r. e. may be explained by changes of molecular state in the bulk liquid. The present results prove that the whole behaviour of $r$. e. previously studied $\left.{ }^{2 a}, \mathrm{~b},{ }^{\circ}\right)$, is due to such changes.

Physical Laboratory of the Polytechnic Institute, Timisoara, Roumania

\section{BoRneAs and I. BǍBuţiA}

Eingegangen am 10. Mai 1960

1) BǍbuȚta, I.: Stưd. şi cerc. şt. chim. Timişoara 6, Nr. 3-4 (1959). - 2) Borneas, M., and I. BẲBuţIA: C. R. Acad. Sci. [Paris] a) $248,1164,2865,3281$ (1959); b) 249,1036 (1959); c) 250,1613 (1960); d) 250, 2330 (1960); e) Stud. si cerc. st. chim. Timişoara 6, Nr. 1-2, 153 (1959); f) Acta phys. polon. 18, 521 (1959). - $\left.{ }^{3}\right)$ BorNEAS, M., and E. KaLMAN: C. R. Acad. Sci. [Paris] a) 245, 1710 (1957); b) 246, 944, 2256 (1958). - 4) Landol T-Börnstein, Zahlwerte und Funktionen, vol. II, part 3. Berlin-Göttingen-Heidelberg: Springer 1957.

\section{Zur Theorie des Bandwalzens}

Seit den Untersuchungen von TH. v. KÁRMÁN ${ }^{1}$ ) und E. Orowas ${ }^{2}$ ) über die plastizitätstheoretischen Grundlagen der Umformvorgänge beim Bandwalzen wurde in zahlreichen
Arbeiten versucht, die angegebenen Lösungen $\left.{ }^{1}\right)^{2}$ ) für die ingenieurmäßige Anwendung zu vereinfachen. Die Forderungen, die sich aus einer derartigen Anwendung für ein Berechnungsverfahren ergeben - Einfachheit und hinreichende Genauigkeit - , sind jedoch nicht leicht miteinander zu vereinbaren. Jedenfalls erfüllen beim Bandwalzen die bekannt gewordenen Verfahren zumindest die eine oder die andere dieser Forderungen nicht.

Ein Ausgleich zwischen den erwähnten Forderungen wird jedoch möglich, wenn die strengen, plastizitätstheoretischen Voraussetzungen soweit berücksichtigt werden, wie es im Hinblick auf ertragbaren Rechenaufwand sinnvoll erscheint, während wesentliche Näherungen auf die Integration der Differentialgleichung $\left.{ }^{1}\right),{ }^{2}$ ) des Bandwalzens beschränkt bleiben.
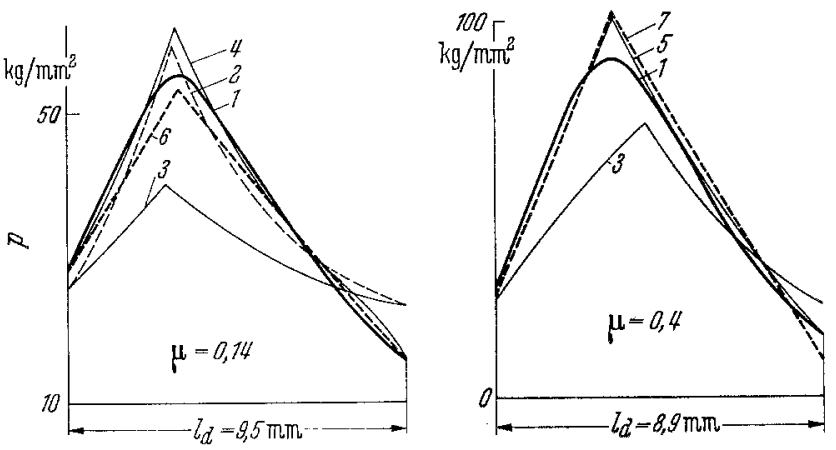

Fig. 1. Verteilung des Normaldrucks $p$ über die gedrückte Länge $l_{d}$. Vergleich zwischen Versuch und Rechnung (Kaltwalzen von Aluminium). 1 Messung von LUEG $^{3}$ ); 2 ber. n. v. KÁRMÁN ${ }^{2}$ ); 3 ber. $n$. SieBEL ${ }^{2}$; 4 ber. n. OrowaN ${ }^{2}$ ) (Gleitreibung); 5 ber. n. OrowaN ${ }^{2}$ ) (Haftreibung); 6 quasilineare Methode (Gleitreibung); 7 quasilineare Methode (Haftreibung)

Über eine der vielen Möglichkeiten für eine derartige Näherungslösung sei hier berichtet. Sie geht von dem Ansatz

$$
p=a_{0}+a_{1} x+a_{2} x^{2}
$$

für die Verteilung des Normaldrucks $p$ zwischen Walze und Band aus ( $x=$ Ortskoordinate in Walzrichtung). Die Koeffizienten $a_{i}$ in Gl. (1) werden durch Einsetzen in die Differentialgleichung des Bandwalzens ermittelt. Dabei ergibt sich, daß $a_{2}$ klein gegenüber $a_{1}$ ist, so daß nach Gl. (1) die Walzenpressung nahezu linear von $x$ abhängt (quasilineare Methode). Die Differentialgleichung kann entsprechend der Annahme des homogenen Stauchens ${ }^{1}$ ) in der Umformzone oder unter Berücksichtigung der Querschnittskrümmung ${ }^{2}$ ) benutzt werden.

Zur Beurteilung der Genauigkeit dieser quasilinearen Methode zeigt Fig. 1 einen Vergleich zwischen gerechneten und gemessenen ${ }^{3}$ ) Verteilungen des Normaldrucks $p$ entlang der Länge $l_{d}$ der Umformzone (Kaltwalzen von Aluminium).

Tabelle. Fließscheidenlage $x_{s}$. Abweichung zwischen den Meßwerten in den 38 von KREULITSCH ${ }^{4}$ untersuchten Fällen und den nach verschiedenen Verfahren berechneten Werten (Warmwalzen von Stahl)

\begin{tabular}{|c|c|c|c|}
\hline \multirow[b]{2}{*}{ Berechnung nach } & \multicolumn{3}{|c|}{ Abweichung vom Meßwert } \\
\hline & $\begin{array}{l}\text { geringer } \\
\text { als } \pm 2 \%\end{array}$ & $\begin{array}{c}\text { größer } \\
\text { als } \pm 10 \%\end{array}$ & größte \\
\hline 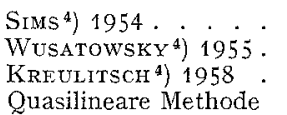 & $\begin{array}{l}\text { in } 21 \% \\
\text { in } 26 \% \\
\text { in } 29 \% \\
\text { in } 31 \%\end{array}$ & $\begin{array}{l}\text { in } 16 \% \\
\text { in } 10 \% \\
\text { in } 10 \% \\
\text { in } 5 \%\end{array}$ & $\begin{array}{l} \pm 16 \% \\
\pm 14 \% \\
\pm 12 \% \\
\pm 12 \%\end{array}$ \\
\hline
\end{tabular}

Für das Warmwalzen von Stahl zeigt die Tabelle Ergebnisse eines Vergleichs zwischen gerechneten und gemessenen ${ }^{4}$ ) Abständen $x_{s}$ der Fließscheide (Ort der höchsten Pressung $p_{\max }$ ) von der Austrittsebene. Der Abstand $x_{s}$ ergibt sich nach der quasilinearen Methode zu

mit den Hilfsgrößen

$$
x_{s}=\frac{1+H_{1} H_{2}}{3+H_{1}}
$$

$$
H_{1}=h_{a} / h_{e} ; \quad H_{2}=1-2 l_{d} / v
$$

( $h_{e}=$ Eintrittsbandstärke, $h_{a}=$ Austrittsbandstärke, $\gamma=$ Walzenradius). 